Continents manuscrits

Génétique des textes littéraires - Afrique, Caraïbe, diaspora

$14 \mid 2020$

Photographie algérienne : de la genèse à la représentation

\title{
Faire collectif
}

Entretien avec Collective220, mené par Rym Khene

\section{Collective220}

\section{OpenEdition}

\section{Journals}

Édition électronique

URL : https://journals.openedition.org/coma/5521

DOI : $10.4000 /$ coma. 5521

ISSN : 2275-1742

Éditeur

Institut des textes \& manuscrits modernes (ITEM)

Référence électronique

Collective220, «Faire collectif », Continents manuscrits [En ligne], 14 | 2020, mis en ligne le 01 avril 2020, consulté le 12 janvier 2023. URL : http://journals.openedition.org/coma/5521 ; DOI : https:// doi.org/10.4000/coma.5521

Ce document a été généré automatiquement le 12 janvier 2023.

\section{(2) $(\mathcal{Q} \Theta \Theta$}

Creative Commons - Attribution - Pas d'Utilisation Commerciale - Pas de Modification 4.0 International - CC BY-NC-ND 4.0

https://creativecommons.org/licenses/by-nc-nd/4.0/ 


\section{Faire collectif}

Entretien avec Collective220, mené par Rym Khene

\section{Collective220}

1 Dans cet entretien mené au printemps 2020, le Collective220 pose un regard rétrospectif sur son parcours et sa pratique de la photographie. Si le collectif existe depuis cinq ans seulement, il est certain qu'il contribue déjà à dynamiser le paysage visuel algérien par les regards neufs et originaux de ses photographes. Nous les avons interrogés sur leur pratique et les défis à relever. 
Figure 1 : Autoportrait du Collective220

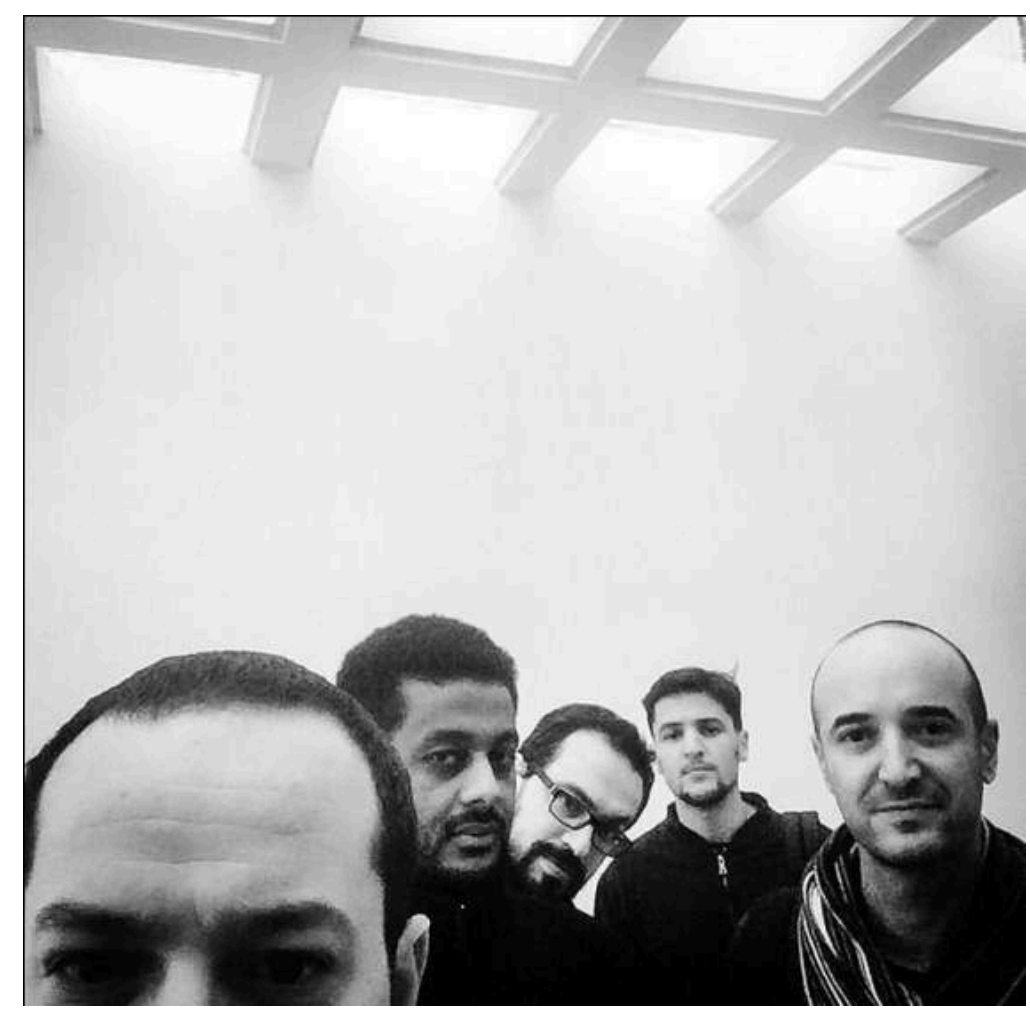

De gauche à droite : Youcef Krache, Abdo Shanan, Houari Bouchenak, Fethi Sahraoui, Ramzy Bensaadi

(C) Collective220 / 2018

RYM KHENE : Comment est né le projet de mettre en commun vos regards et vos expériences?

Collective220 : Collective 220 est né en 2015, dans la chambre 220 de l'hôtel Albert I ${ }^{\text {er }}$ à Alger ${ }^{1}$. Au départ, nous étions un groupe de photographes qui participions au Festival national de la photographie $\left(\mathrm{FesPA}^{2}\right)$. Nous nous étions réunis pour échanger sur nos différentes pratiques et visions de la photographie, et avons décidé, sur place, de former un collectif. Le nombre de photographes a évolué au fil des années et certains d'entre eux, alors qu'ils ont contribué à la mise en place de la structure et des objectifs, ne sont plus des membres actifs du collectif, mais sont toujours présents par leurs conseils et suivis de différents projets. Aujourd'hui, le collectif compte cinq personnes: Ramzy Bensaadi, Houari Bouchenak, Youcef Krache, Fethi Sahraoui et Abdo Shanan.

Nous avons voulu rassembler les énergies créatrices des différents photographes afin de structurer nos actions, clarifier nos démarches et nos regards et, ainsi, préciser les enjeux esthétiques que nous percevions en Algérie. Il y avait aussi un ras-le-bol des clichés et de la manière avec laquelle l'image de l'Algérie est exportée à l'étranger, souvent par les Occidentaux.

Le constat de l'invisibilité de la photographie dans le champ culturel national nous a confrontés à l'obligation de combler ce vide, notamment en multipliant l'accès à la photographie par le biais de différents canaux: ateliers, expositions, festivals, publications, rencontres, débats, projets d'édition... L'ambition du collectif est aussi celle de créer une banque d'images qui servira de repères visuels mais qui sera aussi 
une source d'archives, un vecteur par lequel une histoire contemporaine de la photographie algérienne pourra être élaborée.

RK : Votre travail en tant que collectif, semble offrir une multiplicité de regards sur l'Algérie mais aussi sur le monde.

Collective220 : On n'est jamais objectif par rapport à un sujet, chacun des membres $\mathrm{du}$ collectif a sa propre approche, ses recherches, qui évoluent au fil du temps, naturellement. Nous sommes tous dans une démarche documentaire, mais qui prend pour chacun une forme et une trajectoire singulières.

En ce moment, Abdo Shanan propose des travaux autour des questions de quête identitaire et d'exil avec sa série Dry, réalisée dans le cadre du programme Arab Documentary Photograhy; Fethi Sahraoui est plus porté vers la jeunesse des stades et celles de régions rurales et citadines, tout un monde que l'on pourra bientôt découvrir dans un livre, Triangles de vues $^{3}$, ainsi que dans une pièce multimédia, Youthupia, réalisée en partenariat avec la fondation Magnum. Youcef $\mathrm{Krache}^{4}$, quant à lui, est à la recherche d'esthétiques populaires, avec un souci de rendre visible et tangible la pratique photographique dans la cité. Ramzy Bensaadi ${ }^{5}$ a un côté un peu plus « underground», il offre, avec cette distance bienveillante qui est la sienne, un regard au plus près de la population qu'il rencontre. Enfin, Houari Bouchenak ${ }^{6}$ a une approche plus intimiste avec ses travaux sur le portrait notamment.

Figure 2 : Extrait de la série " Dry »

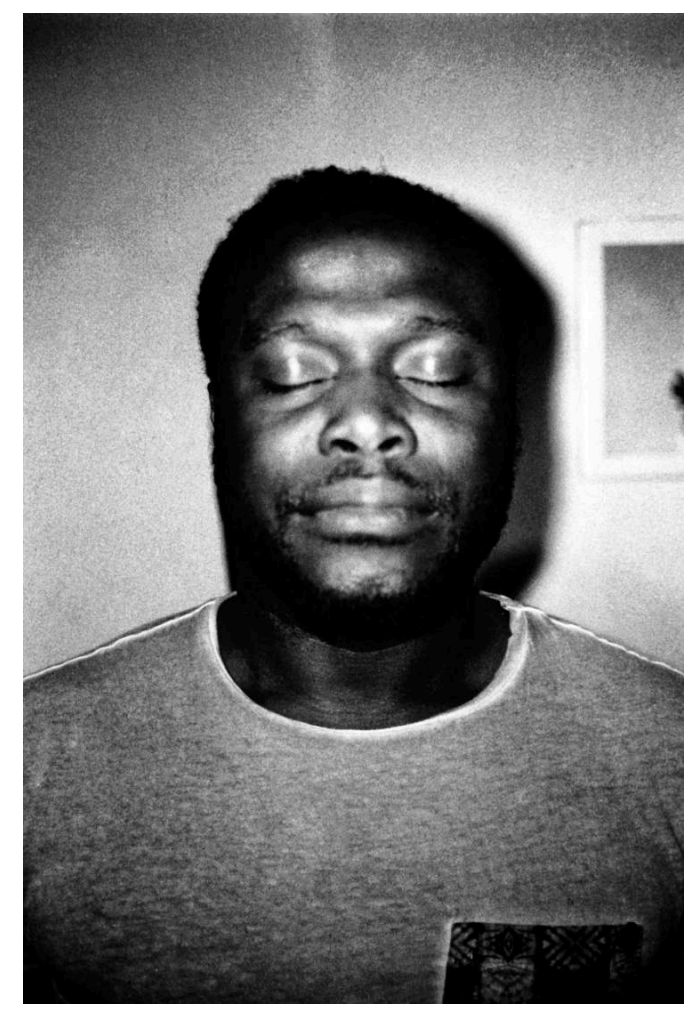

Abdo Shanan

(C) Collective220 / 2017 
Figure 3 : Extrait de la série "Dry »

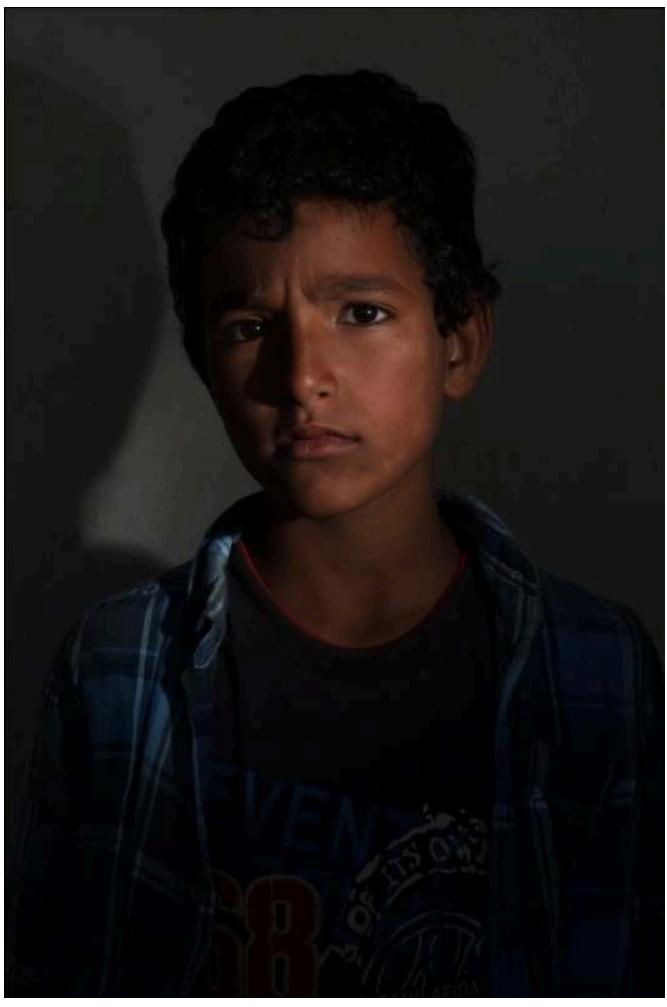

Abdo Shanan

(C) Collective220 / 2018 
Figure 4 : Extrait de le série " $B$ as in Bouchentouf »

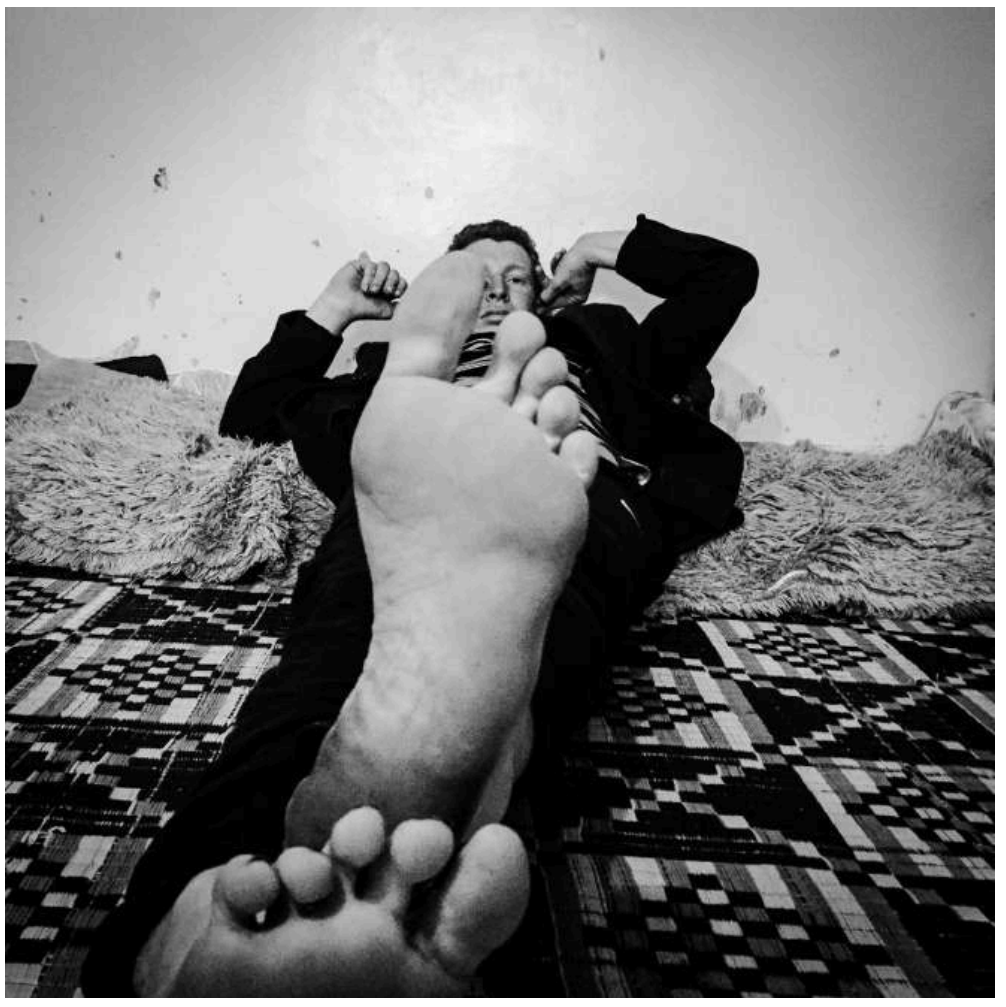

Fethi Sahraoui

(c) Collective220 / 2017 
Figure 5 : Extrait de le série " $B$ as in Bouchentouf »

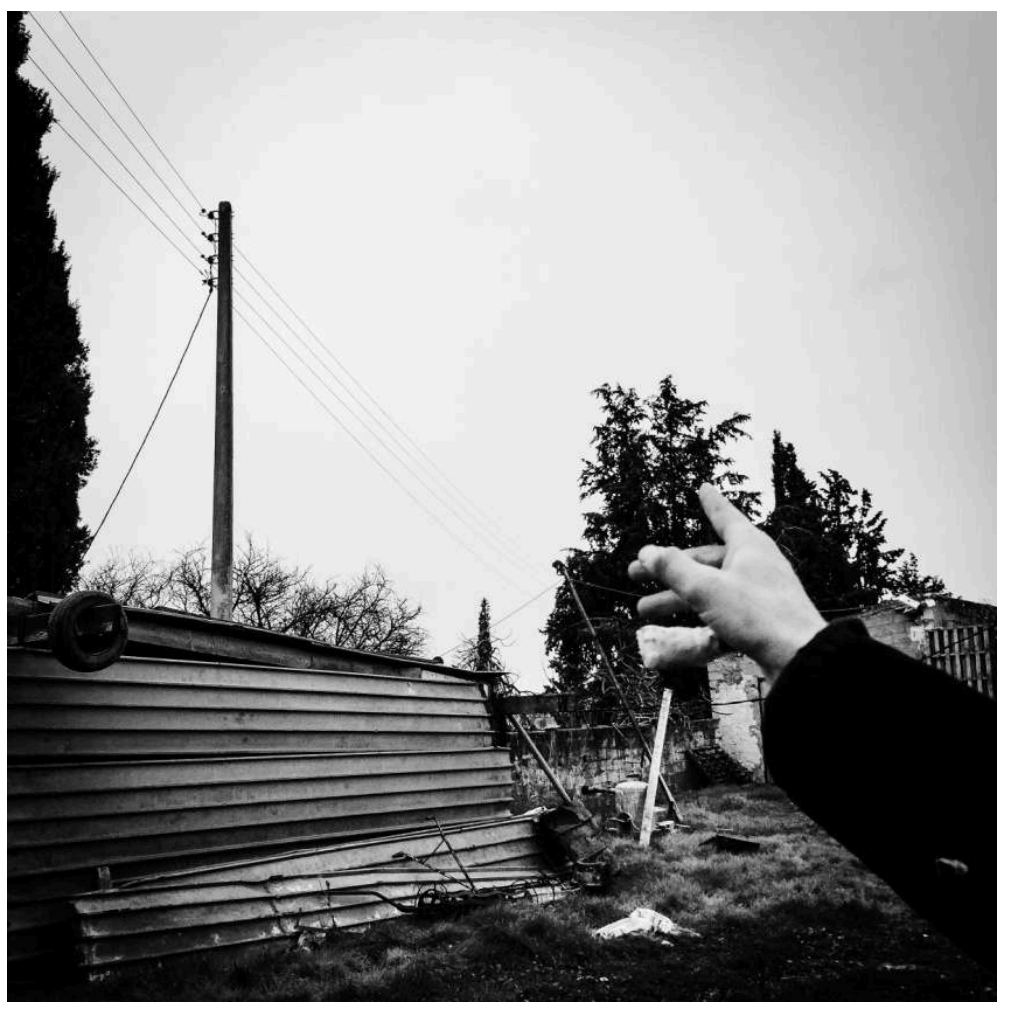

Fethi Sahraoui

(c) Collective220 / 2017

Figure 6 : Extrait de la série "Séoul »

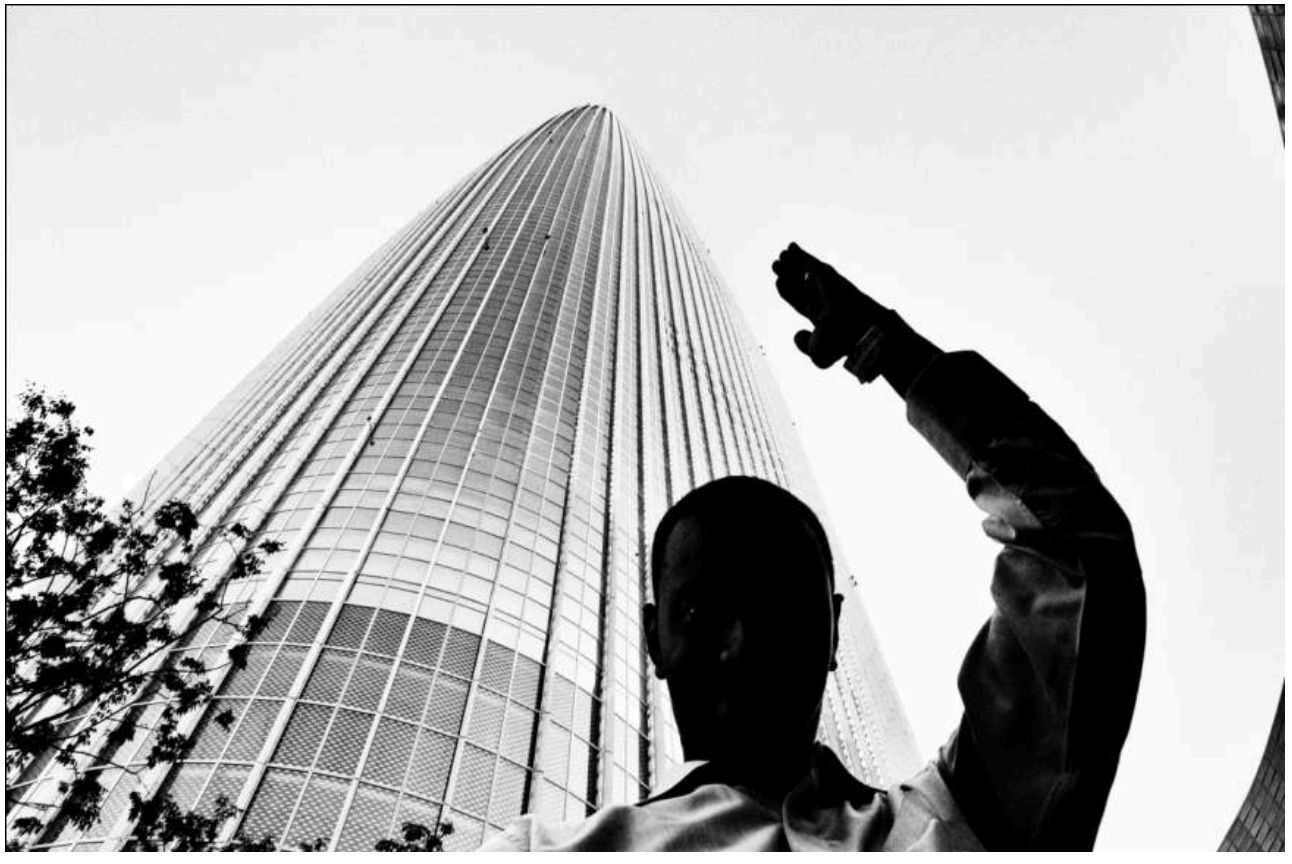

Youcef Krache

(c) Collective220 / 2017 
Figure 7 : Extrait de la série « Séoul »

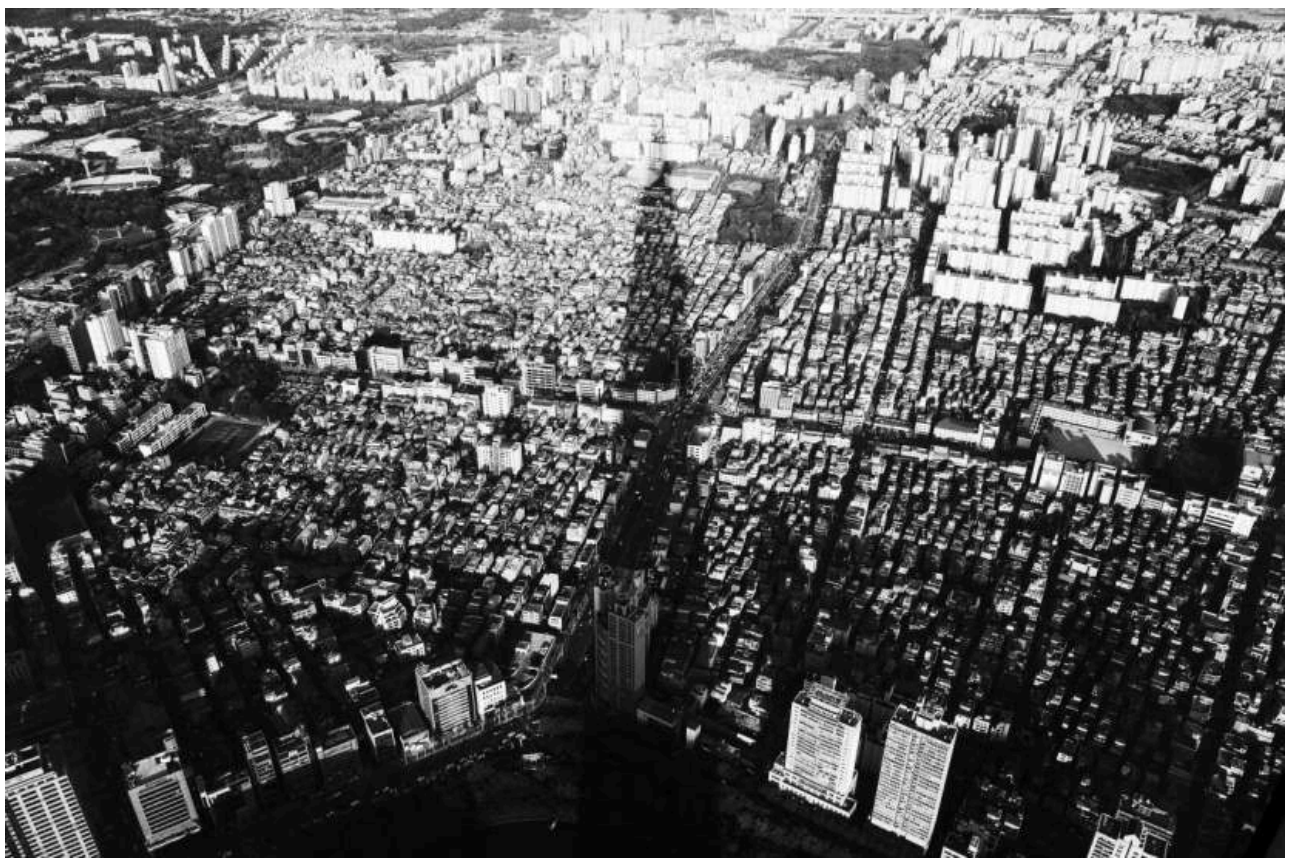

Youcef Krache

(c) Collective220 / 2017

Figure 8 : Extrait de la série « Oran »

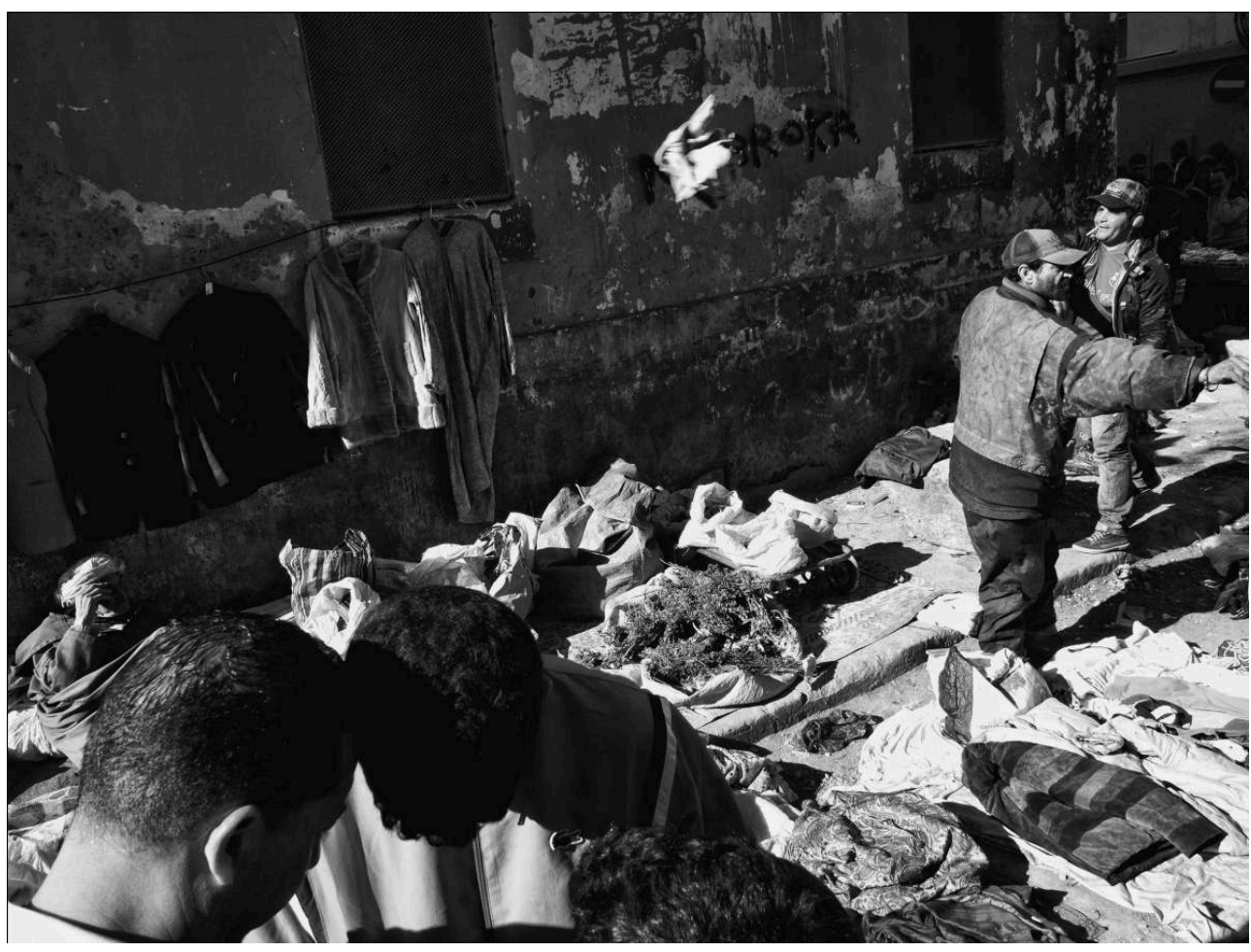

Ramzy Bensaadi

(c) Collective220 / 2014 
Figure 9 : Extrait de la série «Oran »

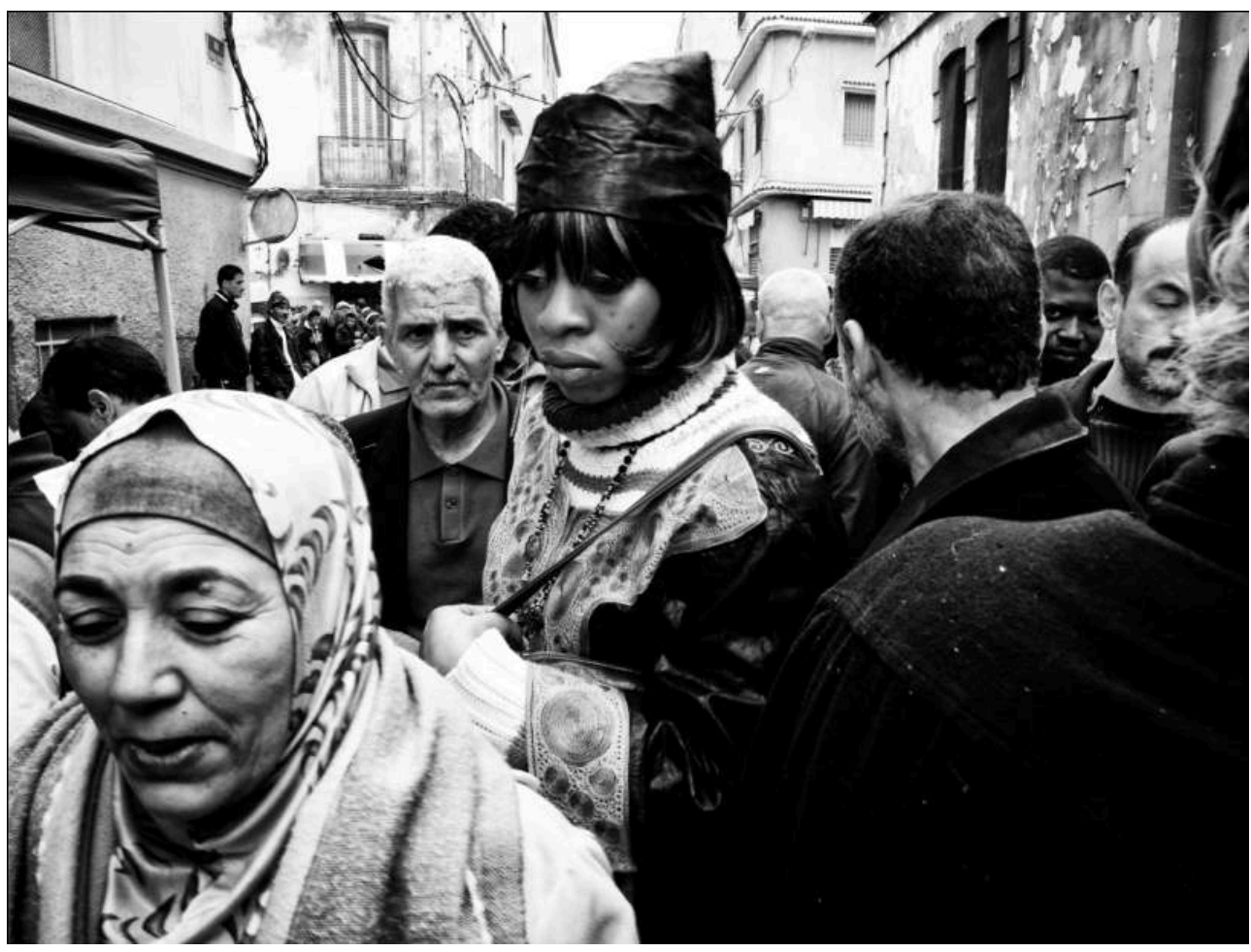

Ramzy Bensaadi

(c) Collective220 / 2014 
Figure 10 : Extrait de la série "Kulturuge »

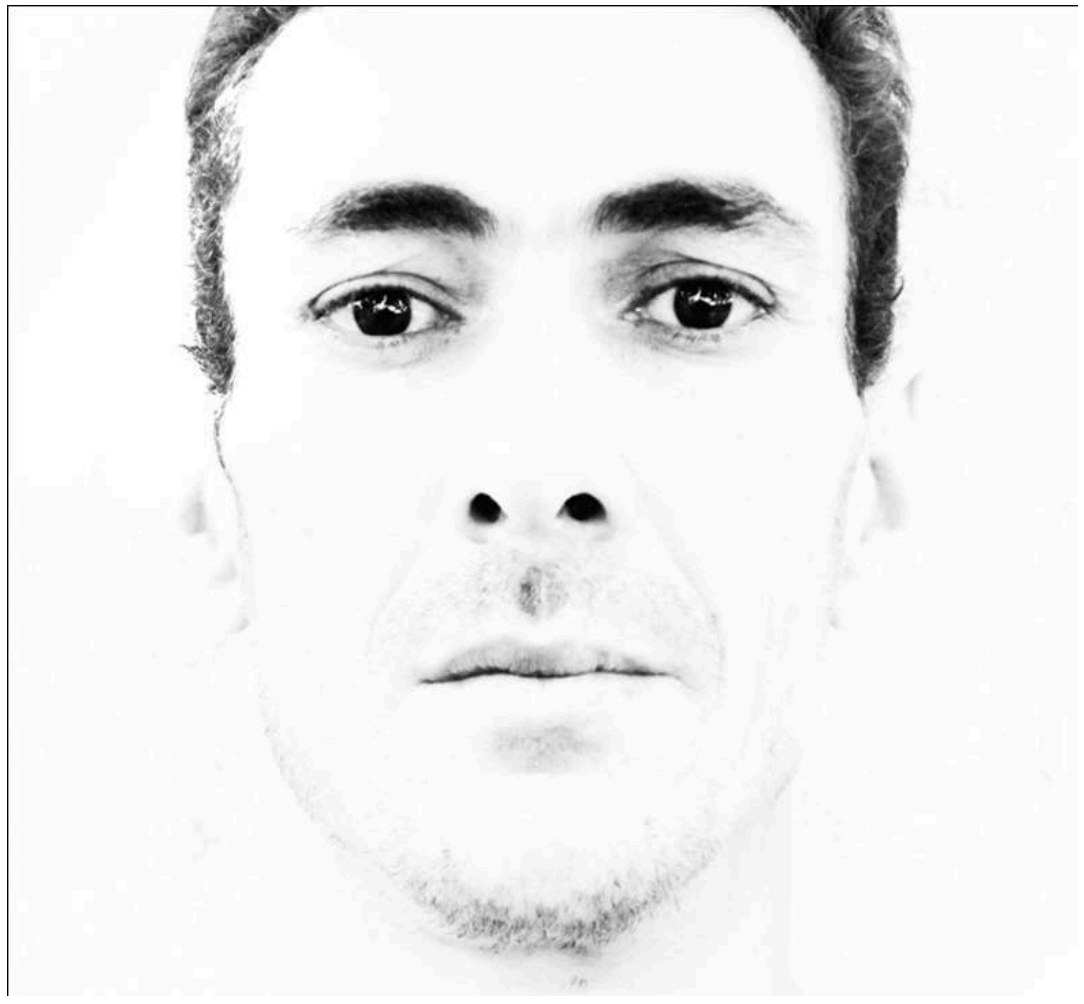

Houari Bouchenak

(c) Collective220 / 2014 


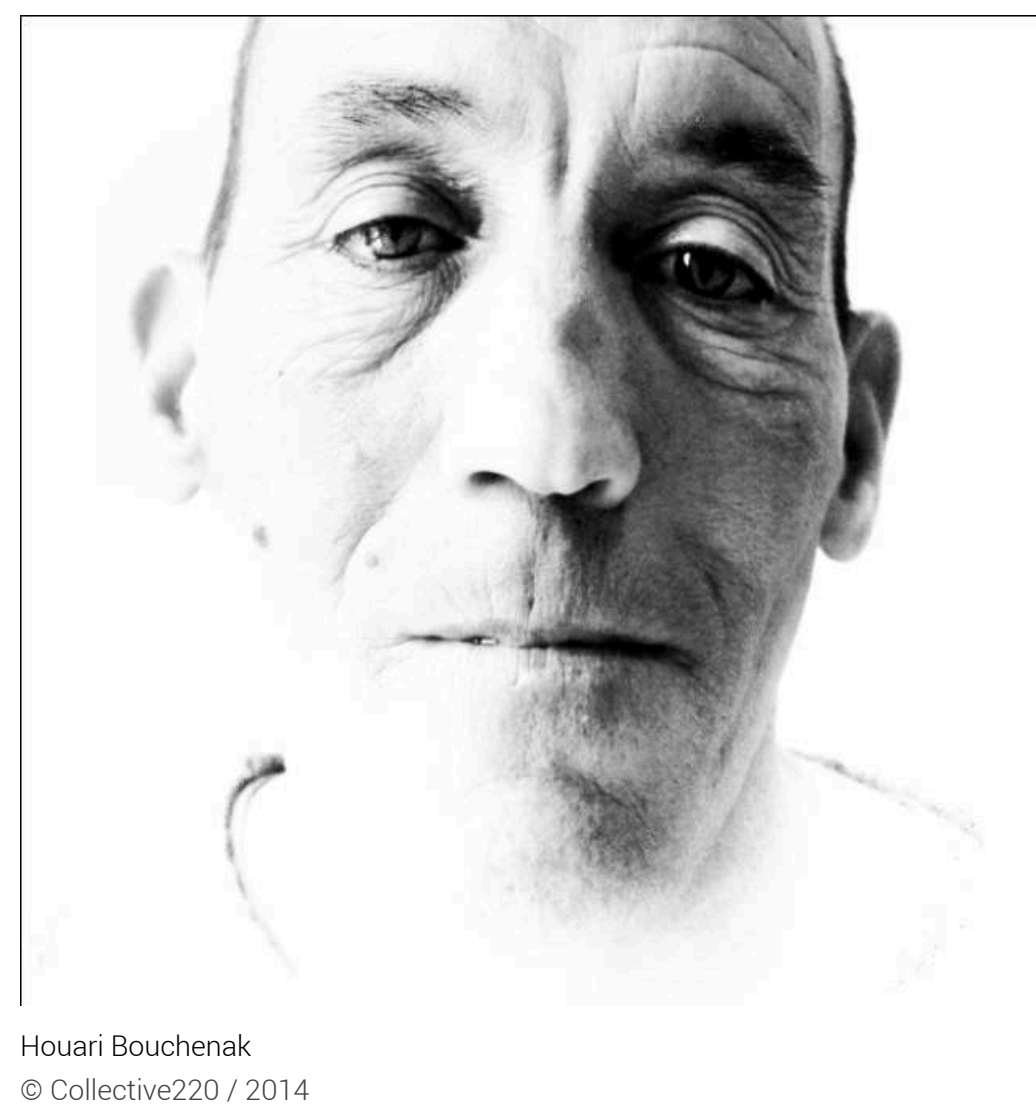

En général, nous sommes dans une perspective documentaire, à la recherche de nouvelles façons de créer des récits bien ancrés dans notre réalité. Et, c'est peut-être la dimension de l'humanité qui anime notre travail à tous, à la fois découvrir et offrir un regard sur les Algériennes et les Algériens tels que nous les voyons et connaissons.

Il y a aussi une dimension importante de notre travail qui consiste à échanger avec d'autres artistes en Afrique et dans le monde, par le biais de festivals et rencontres photographiques notamment. Les dernières années ont été très riches, nous avons par exemple participé aux Rencontres photographiques de Bamako, et aux Rencontres d'Arles sur invitation de l'agence Myop, et nous avons aussi été invités à présenter nos travaux à Barcelone ou à New York. C'est souvent l'occasion d'échanger avec d'autres photographes, d'autres collectifs, ainsi qu'avec le public, et c'est passionnant, particulièrement dans le contexte algérien où la pratique photographique n'a pas de réel statut et où Internet et les réseaux sociaux jouent encore un rôle fondamental dans la communication autour de nos travaux. Finalement, Internet nous a non seulement permis de nous rencontrer, mais aussi de diffuser de nouveaux regards sur un pays qui est trop souvent perçu comme monolithique.

RK : Quel est, selon vous, le rôle de la photographie en Algérie? Quels sont les défis à relever?

Collective220 : En Algérie, la photographie n'a pas encore la place qu'elle mérite. Elle a souvent été un matériau anthropologique ou a eu une fonction purement 
journalistique ou politique. La photographie comme pratique artistique est encore invisible en quelque sorte.

Concrètement, les défis à relever sont de taille. Il y a d'abord, la question du statut du photographe qu'il faut encore préciser puisqu'il y a encore un flou juridique dans lequel le photographe oscille entre la fonction de journaliste et de plasticien. Puis, et cela rejoint le premier point, il y a la question institutionnelle. Il n'existe pas à ce jour de Maison de la photographie, ou de musée exclusivement dédié à la pratique photographique. Comment alors rassembler toutes les initiatives privées pour créer des banques d'images ? Cela arrivera peut-être lorsque le travail de sensibilisation à la photographie, et à l'image en général, sera plus répandu... C'est tout un écosystème qui est à construire.

Youcef Krache par exemple, avec une initiative qu'il a voulue militante, a investi la rue Didouche Mourad dans le centre-ville d'Alger, un matin, le 5 juillet 2015, en collant des centaines d'images sur les murs. Il a utilisé un support simple, des feuilles de format A4, qu'il a collées sur les murs de la ville. La majorité a été décollée dans la journée, mais certaines ont été rescapées, et il y a eu, le temps de quelques jours, des traces photographiques sur les murs d'Alger... L'idée était de délocaliser les rares lieux d'exposition et tenter, à son niveau, de rendre la photographie accessible à tous.

Figure 12 : Collage de photographies de Youcef Krache à Alger

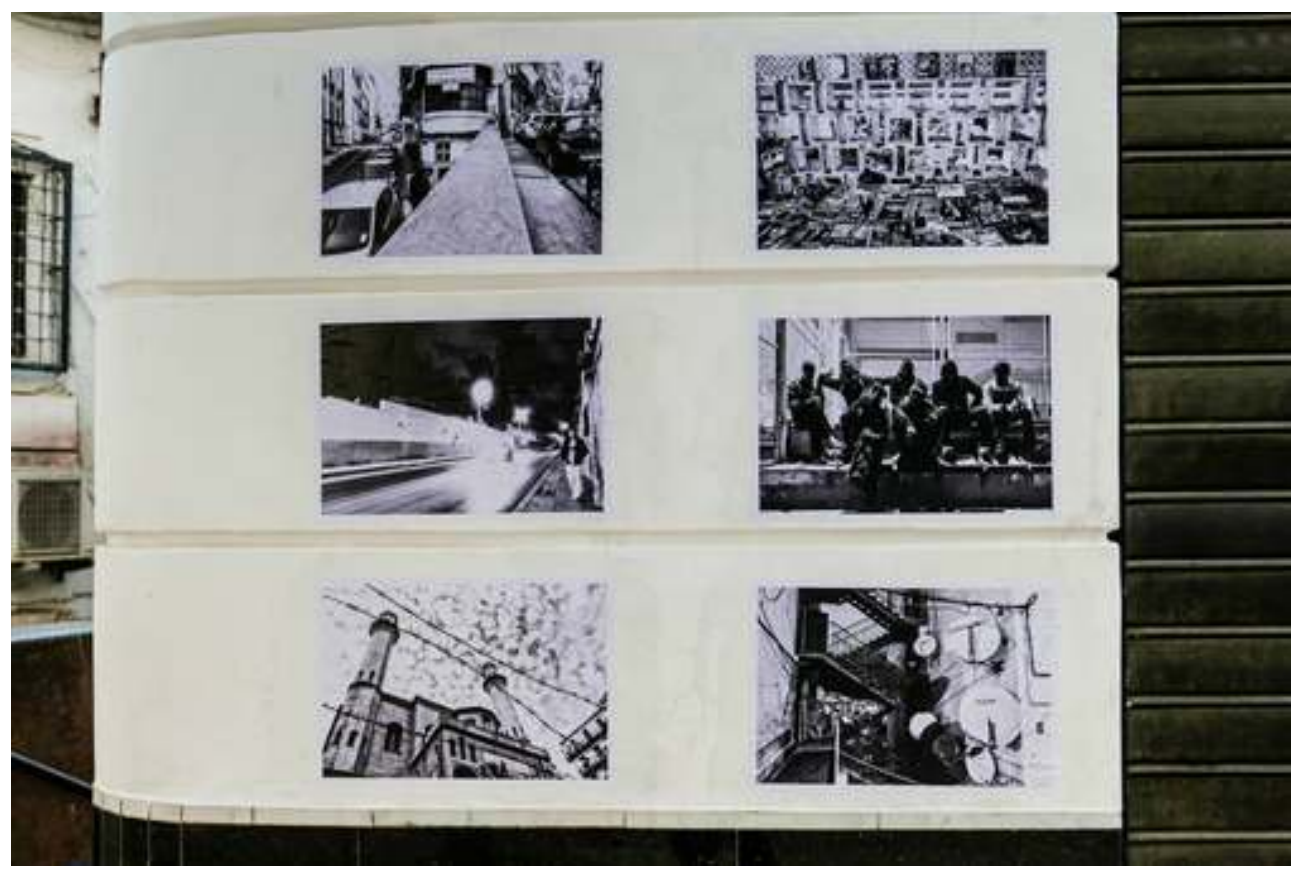

Alger, 5 juillet 2015

(c) Collective220 
Figure 13 : Collage de photographies du Collective220 à Barcelone

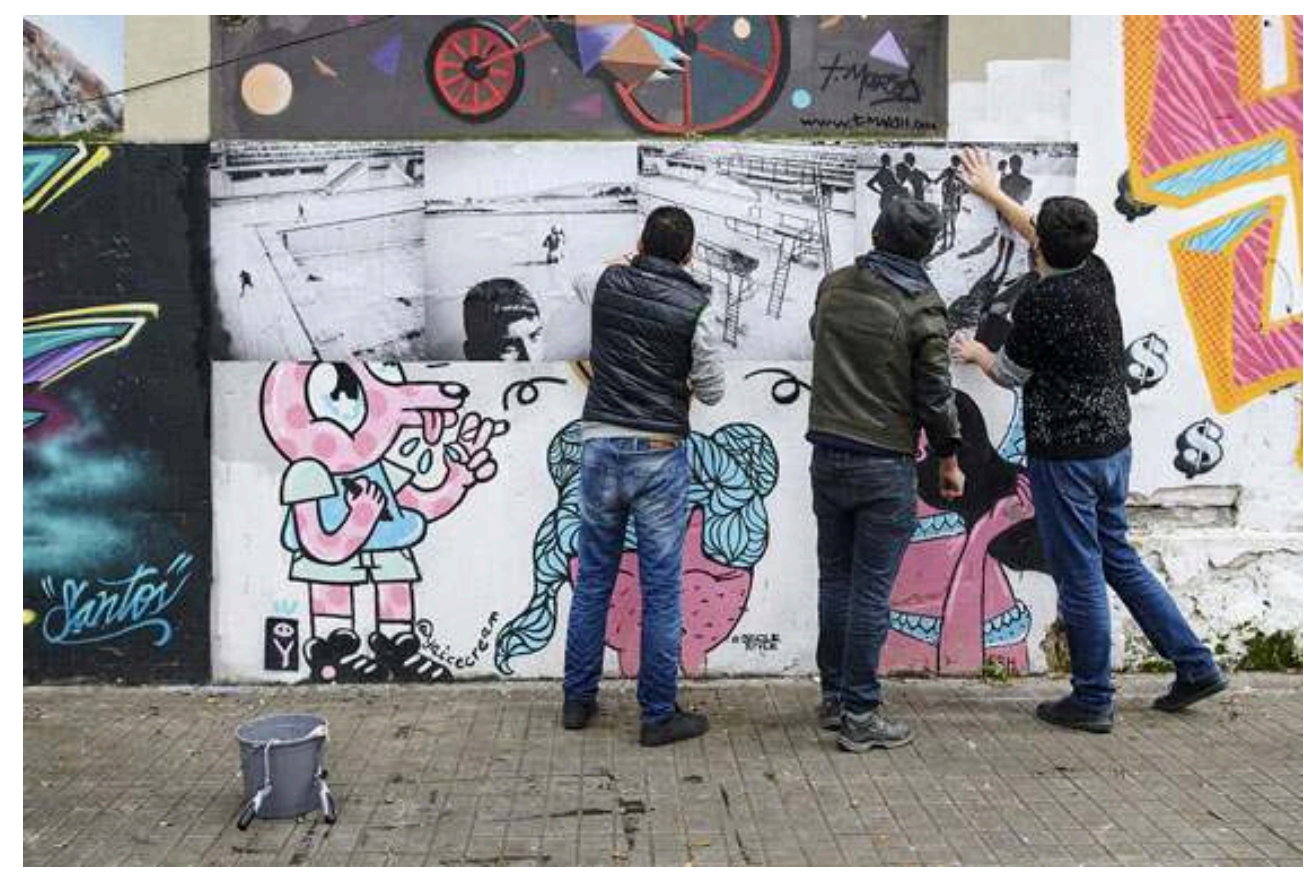

Collage en partenariat avec Jiser, 2018

(c) Éric Pàmies

RK : Comment le manque d'institutionnalisation et de réelle politique culturelle dans le domaine influe-t-il sur votre manière de travailler?

Collective220 : Cela nous oblige à être créatifs, à toujours innover dans nos pratiques et nos démarches. C'est aussi, si l'on veut être optimiste, une liberté dans le sens où nous ne sommes rattachés à aucune institution et restons donc indépendants dans nos regards et nos expérimentations. Par la force des choses, nous sommes autodidactes et cela est peut-être une force finalement. Le rôle de l'artiste est de rendre visible son regard, son opinion, sans les soumettre aux aléas des médias ou des agendas politiques, et c'est ce que nous faisons. Dans cette perspective, nous avons lancé un nouveau festival, Les Rencontres photographiques de Mascara, dont la première édition devait avoir lieu le 19 mars 2020 mais a été reportée au vu de la pandémie du coronavirus actuelle.

De plus, Youcef Krache a fondé La Chambre claire. Soutenue par le collectif, la maison d'édition en est encore à ses débuts, mais elle vient combler un réel vide dans le monde éditorial algérien. Elle est aussi prometteuse dans ce qu'elle propose : une manière nouvelle d'éditer des images, en jouant avec les formats et la mise en page. Mais, ce qui est important aussi, c'est la matérialité-même d'un livre de photographies. C'est fondamental au moment où tout semble être tourné vers le numérique. C'est aussi une manière de faire voyager, rendre accessible, faire corps avec le lecteur.

RK : L'Algérie est un pays qui n'a jamais été étranger à la photographie. Dès le début de la conquête coloniale qui coïncide avec les débuts de la photographie, des codes de représentations ont été élaborés. Les représentations évolueront tout au long de l'histoire, d'abord pendant la guerre d'indépendance ou le FLN mène, comme composante de la guerre, une guerre médiatique, puis pendant les années 1990 où l'image prend encore une 
dimension différente. Quels sont pour vous, les moments ou les figures qui ont marqué ces différentes tranches historiques ? Pourquoi?

Collective220: Les traces photographiques de l'histoire algérienne sont toutes importantes puisqu'au minimum, elles représentent des repères de notre histoire visuelle. En ce sens, il est important d'explorer et d'interroger les travaux de Marc Garanger sur les femmes algériennes par exemple. Le contexte de leur production est connu et controversé, ces femmes qu'il a photographiées dans le cadre de la politique de recensement de la population pendant la guerre d'indépendance, mais il est important de s'interroger, encore aujourd'hui, sur la signification de ces images. Pierre Bourdieu ou Raymond Depardon ont également une production photographique algérienne conséquente qui nous informe sur les luttes visuelles de l'époque. Ce sont autant de traces de la période coloniale à interroger encore aujourd'hui.

Du côté algérien, le travail de Mohammed Dib dans Tlemcen ou les lieux de l'écriture $e^{7}$ est fondamental. Écrivain, et finalement photographe également, il offre dans ce livre un regard singulier sur la ville de Tlemcen avec des photographies prises en 1946 mais aussi sur l'histoire visuelle de ce territoire grâce au texte qu'il écrit en 1993.

Il y a aussi Lazhar Mansouri, photographe qui travaillait essentiellement en studio et qui est encore méconnu. Il a mis en scène nombre d'Algériennes et d'Algériens, dans des postures différentes, qui montrent une joie et une certaine légèreté, caractéristiques des années qui suivent l'indépendance. Dans sa manière de traiter ces images prises en studio nous trouvons les éléments d'une possible tradition photographique algérienne.

Enfin, la décennie 1990 est une période visuellement complexe. Il y a bien sûr La Madone de Bentalha de Hocine Zaourar, lauréat du World Press Photo 1998, qui s'est imposée comme photographie phare de ces années. Mais cette période qui a pourtant été photographiée reste largement invisible ${ }^{8}$.

RK : Quel est, selon vous (ou pour vous) le rôle de l'archive dans ces différents processus?

Collective220 : Ce qui est apparent est que les archives photographiques, toutes périodes confondues, qui sont une source précieuse à la fois pour les chercheurs et pour les artistes, se trouvent essentiellement dans des fonds privés. Il est donc difficile de construire des récits qui sur le temps long. C'est peut-être pour cela que les projets éditoriaux prennent autant de temps à se concrétiser. Le patrimoine visuel existe mais il reste en grande partie inaccessible. Nous ne travaillons donc pas avec des fonds d'archive, mais l'envie y est.

RK : La pratique de la photographie change-t-elle à partir du 22 février 2019 ?

Collective220 : Le 22 février a marqué un vrai bouleversement pour la pratique de la photographie en Algérie. Nous nous sommes spontanément, chacun de son côté, donné l'obligation de couvrir ces événements. Rendre visible ce qu'il se passe, de manière subjective, et couvrir un maximum les marches hebdomadaires citoyennes $\mathrm{du}$ vendredi ainsi que les marches estudiantines du mardi. L'une des forces du collectif est que chaque membre habite une région différente du pays. Nous sommes donc présents dans plusieurs villes, et nous avons ainsi pu rassembler une sorte de panorama des manifestations du mouvement populaire.

Ce qui était délicat, c'est que nous ne sommes pas dans une démarche journalistique, mais documentaire, et nous avons donc l'habitude de travailler sur un temps plus ou 
moins long. Or, depuis un an, il fallait parfois construire des récits sur des temps très courts. Notre manière de travailler a changé. Nous communiquions quotidiennement nos images et nos impressions. Cette révolution nous a aussi renvoyé aux travaux faits par nos prédécesseurs pendant les années 1990. Nous voulions examiner comment différents événements avaient été couverts, tout en étant toujours dans l'actualité mouvementée de cette année pendant laquelle nous nous demandions lesquelles de nos photographies diffuser, publier, et dans quels médias, et lesquelles garder pour plus tard.

Nous avons publié un premier dossier dans Le Monde, et par la suite, dans d'autres organes de presse tels que Libération, le Washington Post ou encore le New York Times.

Notre participation à Marcher !', livre paru en mai 2019, a été également l'occasion de publier en Algérie des photographies de ce mouvement, de poser certaines traces, impressions, images en temps réel. Cela a aussi été une manière de créer une archive, un matériau tangible, esthétique et documentaire du mouvement populaire.

Certains d'entre nous ont également fait le choix de mettre en accès libre sur les réseaux sociaux de nombreuses photographies et de participer au mouvement de la population qui d'une manière ou d'une autre commençait à s'approprier - à prendre en main -, avec un appareil ou un téléphone, la photographie. Pour la première fois, le photographe a eu une place entière - ce qui n'était pas sans risque non plus puisque la répression est devenue une nouvelle fois une réalité -, dans la rue et dans l'espace public.

Le 22 février, c'est aussi un nouvel espace qui s'est ouvert à la photographie.

Figure 14 : Sans titre

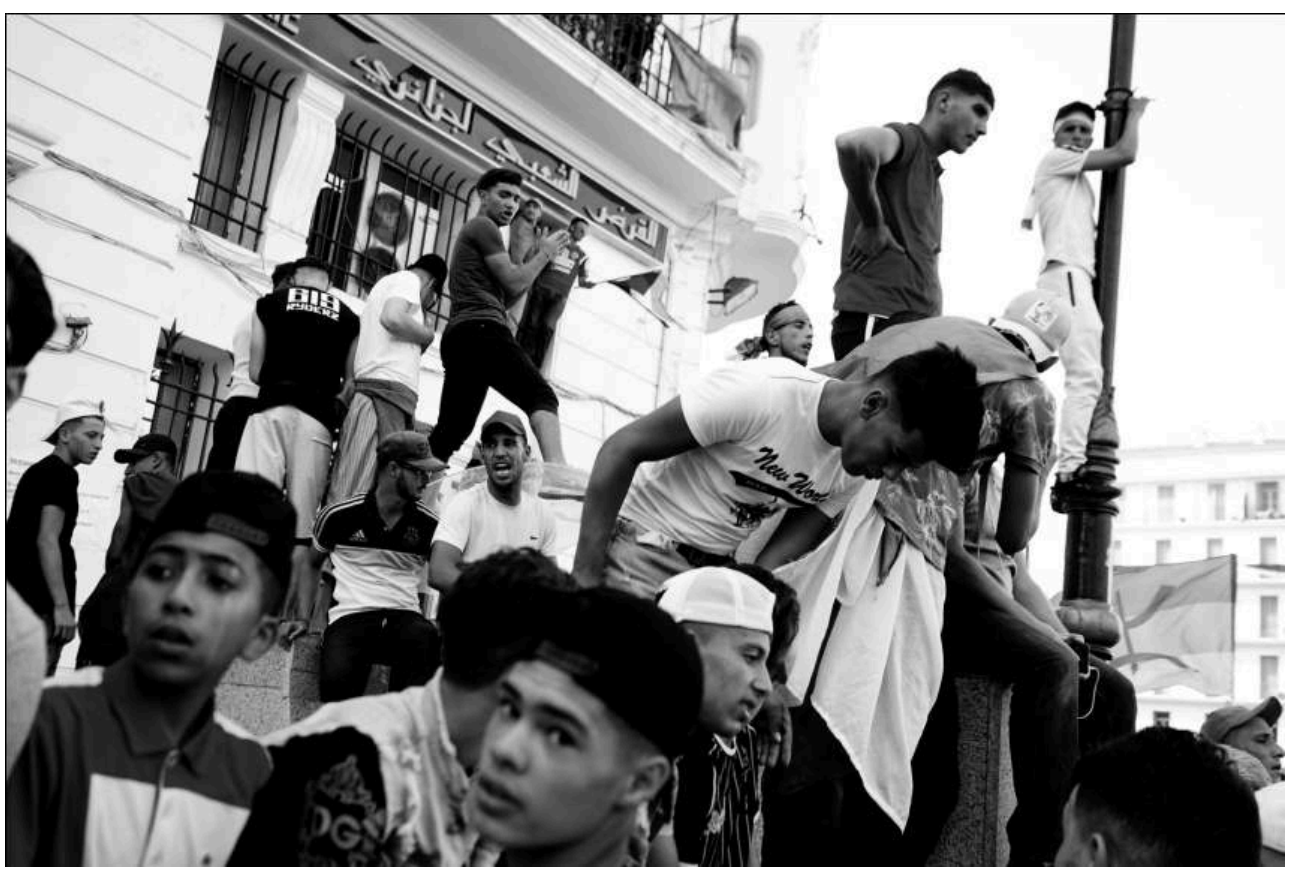

Houari Bouchenak

(c) Collective220 
Figure 15 : Extrait de la série «A little louder »

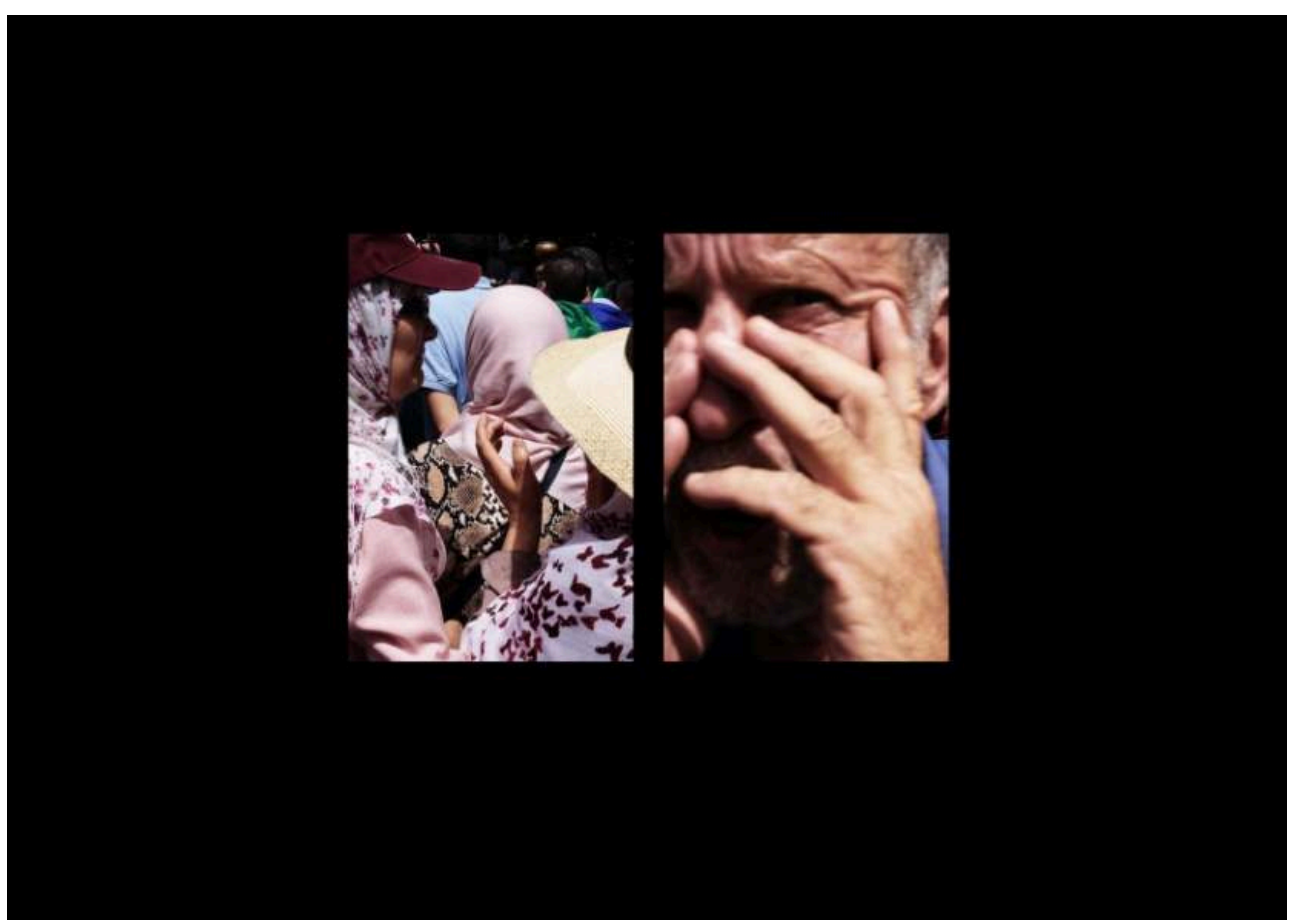

Abdo Shanan

(c) Collective220

Figure 16 : Sans titre

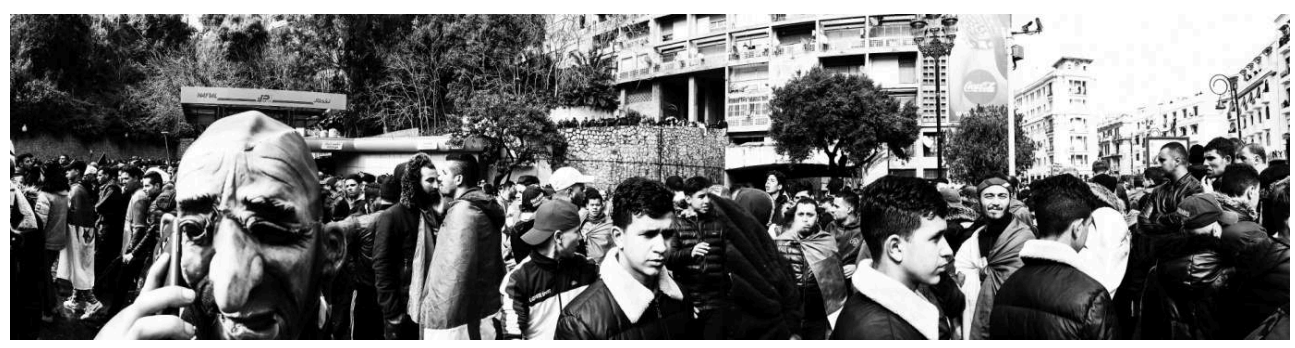

Youcef Krache

(C) Collective220 
Figure 17 : Sans titre

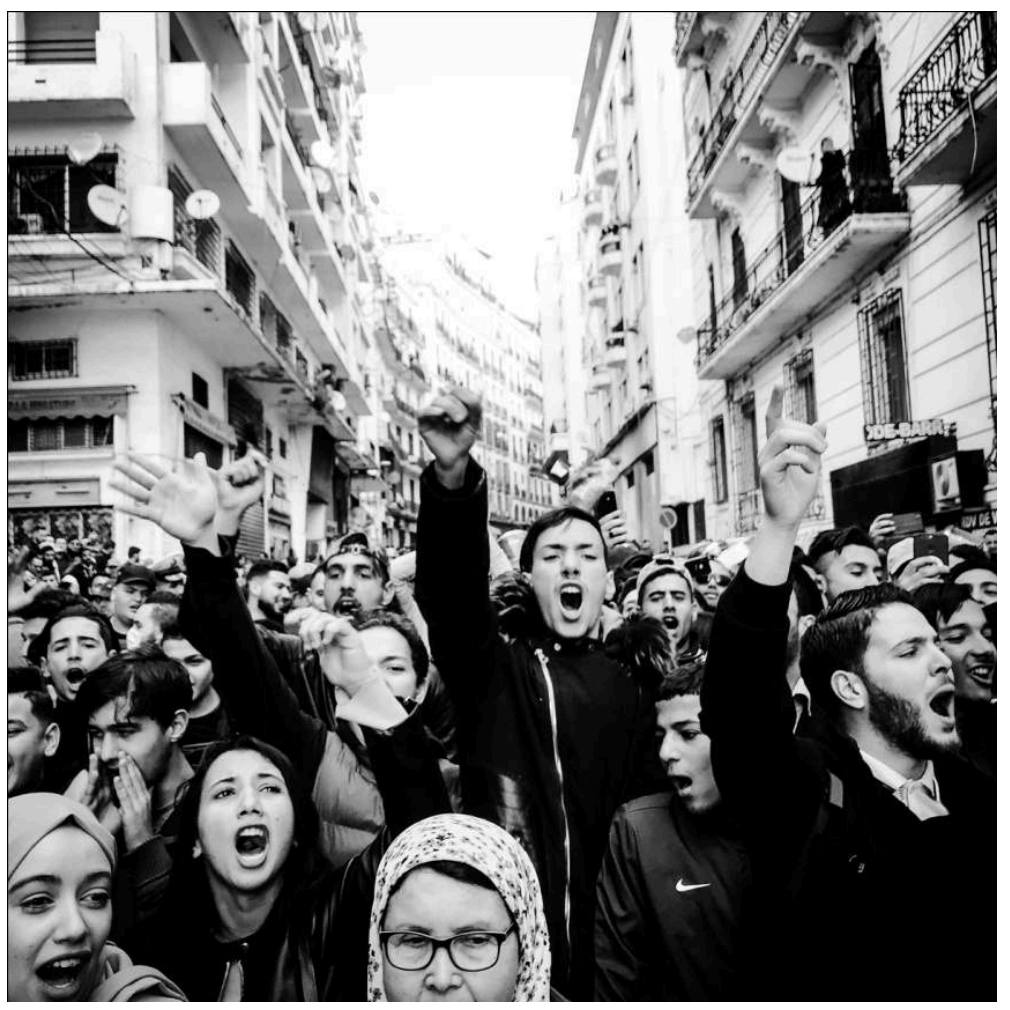

Fethi Sahraoui

(c) Collective220

Figure 18 : Sans titre

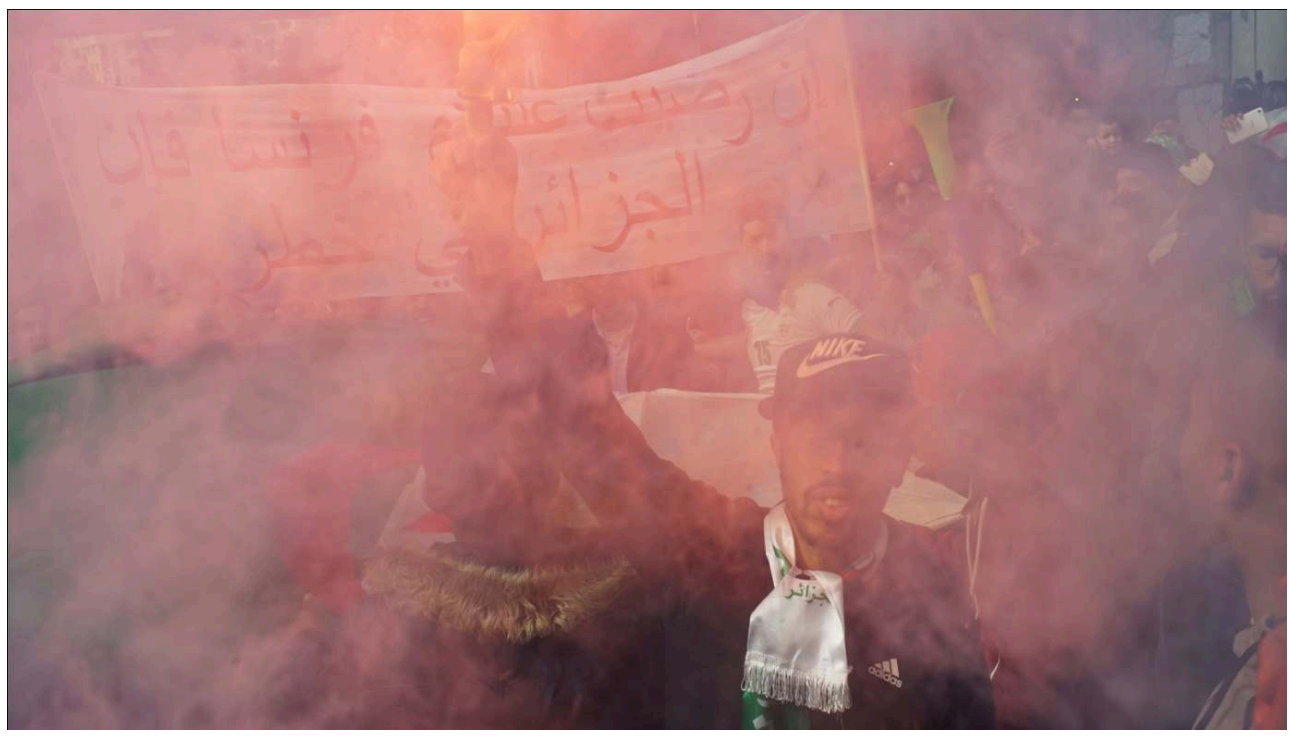

Ramzy Bensaadi

(c) Collective220 


\section{NOTES}

1. Lors de ce premier rendez-vous, étaient présents : Yacine Bellahsene, Arslane Bestaoui, Houari Bouchenak, Youcef Krache, Fayçal Rezkallah et Abdo Shanan. Les débuts du collectif ont également, par moments, rassemblé, Awel Haouati, Sonia Merabet et Naima Kaddour. Fethi Sahraoui et Ramzy Bensaadi, aujourd'hui membres du collectif l'ont officiellement rejoint plus tard mais faisaient partie des initiateurs du projet.

2. Le FesPA (Festival national de la Photographie d'Art) existe depuis 2010 et est hébergé par le Musée d'Art Moderne d'Alger (MAMA).

3. Fethi Sahraoui, Triangles de vues, Alger, La chambre claire, à paraître.

4. Fondateur de la maison d'édition La Chambre claire.

5. Régulièrement publié par Le Monde. Voir par exemple, en Une : https://www.lemonde.fr/ afrique/visuel/2014/04/10/l-algerie-sur-le-vif-par-ramzy-bensaadi_4398730_3212.html

6. Fondateur de La Maison de la photo de Tlemcen en collaboration avec l'Association La Grande Maison.

7. DIB Mohammed, Tlemcen ou les lieux de l'écriture, Paris, Éditions de La Revue noire, 1994. À paraître aux Éditions Barzakh, Alger, en 2020.

8. Voir notamment Ammar Bouras, Algérie, chronique photographique, Alger, Barzakh, 2018 ; et Michael Von Graffenried, Inside Algeria, Aperture, 1998.

9. Marcher! Nous Autres, éléments pour un manifeste de l'Algérie heureuse, dir. Amin Khan, Alger, Éditions Chihab, 2019.

\section{INDEX}

Mots-clés : collectif, photographie, Algérie

Keywords : Collective, Photography, Algeria

Index géographique : Algérie 